\title{
Moon as a Giant Detector for Neutrino Streams from Pulsars
}

\author{
Oleg Borisovich Khavroshkin, Vladislav Vladimirovich Tsyplakov
}

Institute of Physics of the Earth, Russian Academy of Sciences, Moscow, Russia

\section{Email address:}

khavole@mail.ru (O. B. Khavroshkin)

\section{To cite this article:}

Oleg Borisovich Khavroshkin, Vladislav Vladimirovich Tsyplakov. Moon as a Giant Detector for Neutrino Streams from Pulsars. International Journal of Astrophysics and Space Science. Vol. 5, No. 6, 2017, pp. 85-99. doi: 10.11648/j.ijass.20170506.11

Received: November 6, 2017; Accepted: January 15, 2018; Published: January 29, 2018

\begin{abstract}
Unaddressed existence of extraterrestrial sources of high energy neutrinos was discovered in $2013 \mathrm{y}$. But the most promising addition to supernovae includes objects such as pulsars and closes multiple systems. Relying mainly on its own experience in the search and registration of the seismic response at frequencies of pulsars and close binary stars on Earth and the Moon and using the results to detect solar neutrinos work on the study of modulated neutrino fluxes were continued. The study of the spectra of lunar seismic noise revealed a number of significant spectral peaks that match the frequency to 3-4 significant figures with the frequencies of sending energy pulses from pulsars. At the same time availability of registration of seismic peaks and their characteristics are strongly associated with the shape and depth of the radioactive geological structures of the lithosphere of the Moon. That is the energy of interaction modulated beam of neutrinos from the pulsar radioactive elements Moon differently converted into energy of seismic noise. There have also been recorded seismic peaks well coincides with the period of close binary stars. Some of the peaks previously were registered not only as a purely seismic frequency, but as the frequency inherent to radioactive laboratory sources. It is further confirms their astrophysical neutrino origin and similar search becomes the part of high-energy neutrino astronomy.
\end{abstract}

Keywords: Solar Neutrino Stream, ANRI-Absorption, Free Solar Oscillation, Neutrino Stream from Pulsars, Radioactivity Lunar Zones

\section{Introduction}

On the Earth, some effects and visible manifestations or effects of cosmogonic and astrophysical nature, so-called exo signals, were known since ancient times. So far, in the Earth geophysics attention of researchers basically comes down to finding the spectral components in seismic and seismic acoustic noise of the Earth which coincides with the frequency of known frequencies sending signals from pulsars [1-3].

According to Nakamura's catalog (lunar seismicity) and simple assessments of the acoustic (seismic) half-wave response (the lunar surface) on $\gamma$ - and Re-regular pulse radiation $[4,5]$ was presented to the general power of external influence not only on the Moon and Earth. It was investigated the primary acoustic response of the Earth's atmosphere on the impact signal from the cosmological and astrophysical objects and processes particularly from pulsars [6-8]. Some types of young pulsars have a shell - a plasma gas-dust environment, the passage through which modulated at a frequency of rotation of the pulsar radiation (especially Re- and $\gamma$ - range) form a jet of matter. The subsequent reaction of the modulated beam as Re- and $\gamma$ - pulses to the atmosphere and the Earth's surface generates regular seismic signals. The cross section for the capture of power by 40 orders of magnitude greater than the gravitational-wave interaction, allowing a different interpretation of results [1-3] and in the Egyptian expedition confirm these findings and to register a manifestation of high pulsars, for example, PSRJ 0514- 4002A (200, 37Gts ) [6,9]. Mismatch of the different velocities of propagation frequency pulses and pulsar wind optic - acoustic origin are responsible for the complex shape of the peaks, and portions of the spectrum with a constant component. These features are likely to be associated with an incomplete representation of all types of astrophysical effects and especially the existence of the modulated flux of neutrinos from the pulsar accordance to Spiring [10]. 


\section{From the Sun to the Galactic Neutrino Astronomy}

Sources of neutrinos in the solar system are quite diverse from the bowels of the heavenly bodies (planets) to the sun. Therefore, the practical basis of modern solar neutrino astronomy should include series of experimental works, which reliably studied some properties of solar neutrinos and their interaction with the radioactive substance the Sun and Earth, as well as other methods of influence on the radioactivity of [11-15]. This interaction named as anomaly neutrino radio activity absorption (affect). A new page of neutrino astronomy of the Sun - it experiments to monitor the possible impact of natural radioactivity of the Sun carried out by measuring the variation of gamma radiation sample of ore from the Trans-Baikal uranium deposit, characteristics of the sample [11]. Thus it was obtained and analyzed data from the temporary monitoring of radioactive ore sample. Spectral analysis of the resulting implementation reveal hidden periodicity in radioactivity, coinciding with the period of natural oscillations of the sun. In the same period of the oscillations observed extra-long fluctuations in the Earth, which has led to an understanding of the role of the solar neutrino geophysical periodicity and another component (communication) Sun - Earth. But if the modulated flux of solar neutrinos can cause fluctuations in the Earth's solar oscillation periods, the same stream from the pulsar frequencies cause vibrations in the crust of the Earth (or Moon). The mechanism of capture of solar neutrinos, are in a state of near-critical heavy radioactive nuclei. Thus, the observed effects are included in the mechanisms of interaction of the Earth - the sun and the influence on seismicity. Moreover, the marked seismic, geophysical and nuclear-geophysical effects were observed previously [1623], and the strong interactions of solar neutrinos with radioactive nuclei grounded theory [24]. Thus, all of the above, if you are not fully developed neutrino astronomy of the solar system, but also a platform for working with galactic sources. By galactic source of modulated neutrino sources primarily include supernova explosions with a huge component of neutrinos (energy $5-50 \mathrm{MeV}$ ) and the rapidly rotating pulsar whose magnetic fields accelerate charged particles[10]. In structure, regardless of pulsars have a speed as the planet's crust, but because of the nuclear matter. The loss of mass of the pulsar radiation field is accompanied by a restructuring of the crust, accompanied by the release streams of particles with relativistic velocity around and their subsequent interaction with the shell of the pulsar and interstellar matter. Therefore, regardless of the pulsar can be seen as a source of modulated neutrino flux. In addition to the sources of neutrinos are quasars, binary stars, and X-ray binary systems of neutron stars, Gamma - ray flashes; promising source - gamma radiation in the $\mathrm{TeV}$ - a range of Crab Nebula. Neutrinos are able to leave the dense medium, for example, the central zone of the Sun, and thus be a tool for the study of previously inaccessible areas and processes. E. The installation with the sensing elements in the form of radioactive substances may be part of the telescopes for recording not only galactic objects, but also a kind of probes for the study of astrophysical objects [11-15]. Close analogue of such plants can be the type of geophysical system, which has a radioactive sensor are geological structures enriched radioactive minerals and the mass of many orders of magnitude superior to cubic kilometers of water existing neutrino telescopes [10]. The registration system of the telescope is a seismic network or a single geophone. Variable neutrino flux interacting with radioactive geological structures, in addition to changes in heat flow that quickly offset will cause the seismic oscillations of these structures recorded seismic network [12- 14; 16 - 23]. The optimal geophysical telescope is a lunar expedition Apollo seismic network and the seismic catalog of the network (Catalogue Nakamura), previously served as a rich source of information, both on the internal structure of the Moon and other objects in the solar system $[6,25]$. The effectiveness of neutrino geophysical telescope (see. Figure 1) is determined by the content of radioactive elements (indirectly, but the magnitude of the correct heat flow) in geological structures beneath the seismic station in particular and / or near the adjacent and underlying structures.

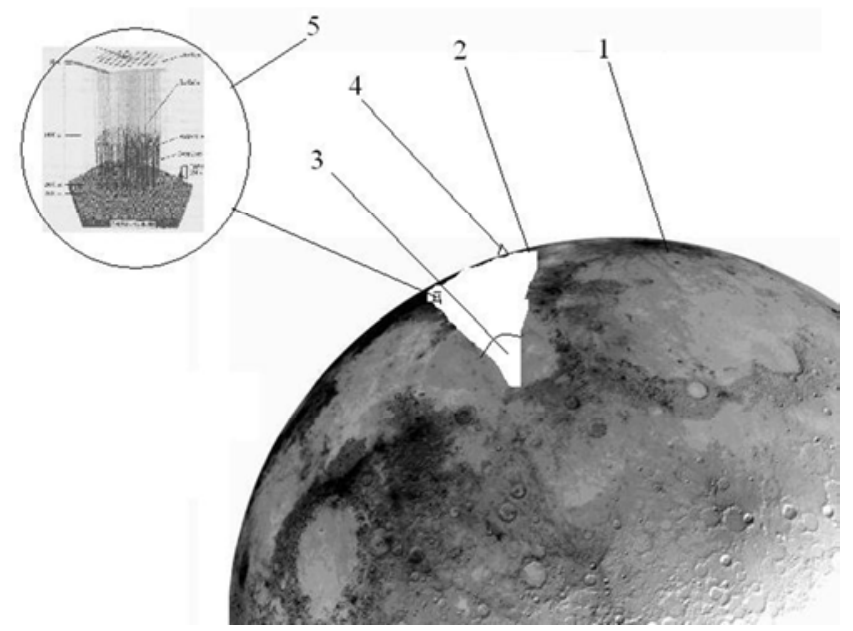

Figure 1. Schematic diagram of the geophysical neutrino telescope on radioactive rocks on the Moon: The moon 1-, 2- surface radioactive deposit, 3 - deep reservoir, 4 - seismic, 5 - $\mathrm{km}^{3}$ icy detector [10].

Luckily Apollo astronauts' expeditions 15 and 17 carried out measurements of heat fluxes at the landing site, and the further development of the picture according to the Lunar Prospector spacecraft is shown in [25], ris. 2, 3. These missions Apollo 15 and 17 cause a lot of interpretation and discussion, which did not influence their role in the neutrino detector in addition to the proximity to the border Lunar Sea (Figure 2). Data on the concentration of Th in the surface structures of the lunar surface, measured the spacecraft Lunar Prospector; visible region of high concentrations of Th around and south of the Sea of Rains (Figure 3). Remote measurement (sensing) of the concentration of heat generating elements $(\mathrm{U}, \mathrm{Th}, \mathrm{K})$ on the surface of the Moon were shown in this area (Figure $2 \mathrm{March}$ ). The question remains - is the surface layout As for the underlying layer, or 
these elements are really focused in the mantle. In part, this question can answer structure seismic response at frequencies of pulsars, the Apollo seismic network recorded in the region of the location of the network (Figure 2,3). Upon registration of the seismic response to a periodic neutrino flux from the pulsar seismic station located in the zone of radioactive structures are structures in the surface of the lunar crust, we

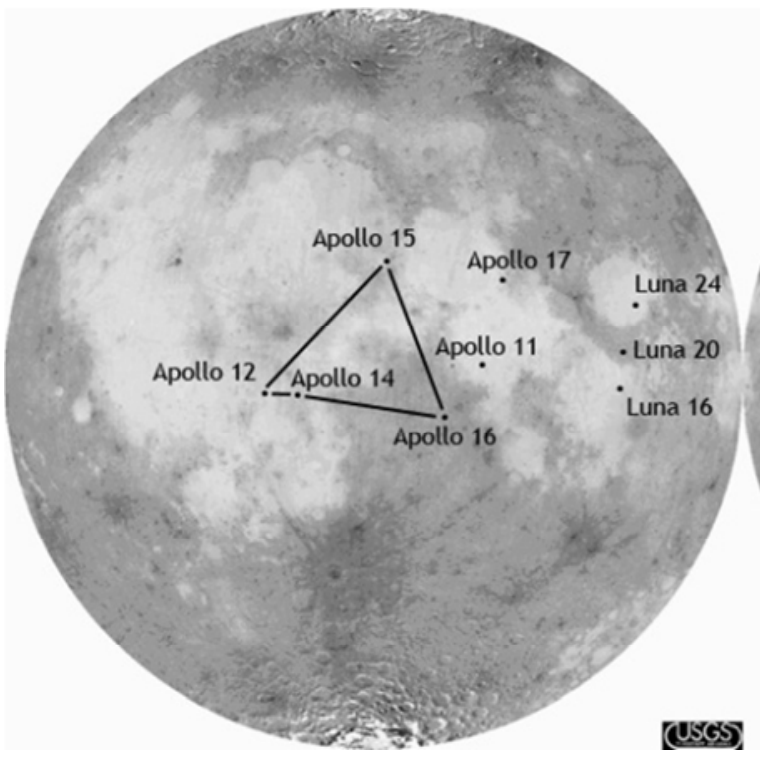

should expect strong signals on the surface $\mathrm{X}, \mathrm{Y}$ - and components weakened and noisy in the Z- component. When placing radioactive species is not only in the surface layers of the cortex but also in the seismic response of the mantle should be primarily powerful Z- component. The latter case is more likely for Apollo 15 seismic region with maximum heat flux landing about $21+3 \mathrm{mWm} 3$ (Figure 3).

Figure 2. The location and plan the Apollo seismic network (approximately equilateral triangle with sides of $\sim 1200 \mathrm{~km}$ ); Left side of the Moon.

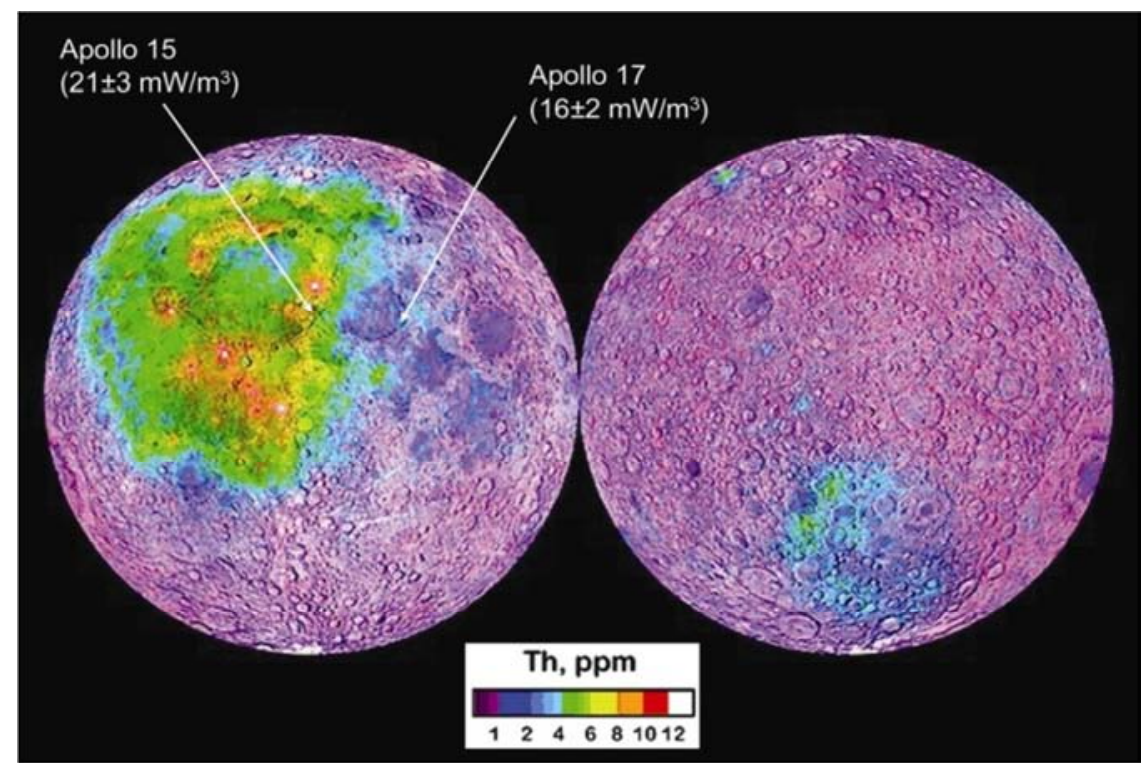

Figure 3. The concentration of Th in the surface structures of the lunar surface; in places of landing Apollo 15 and 17 heat flow measurement indicated numerically.

\section{There Is Analysis of Seismic Noise and Signals in the Region of the Moon Apollo Seismic Network, the Search for Manifestations of Pulsars}

In the analysis of lunar seismic noise records and event records selected areas without major seismic disturbances to the processing of previously received seismograms [26]. Comparative analysis has shown that the best records that contain the frequency at speeds of pulsars obtained seismic Apollo 15. The maximum frequency response of the seismometer is in the frequency range from 0.35 to $0.73 \mathrm{Gts}$. Figure 4 shows a sample direct Z-component seismic data noise, and in Figure 5 - the energy spectrum of the record. 


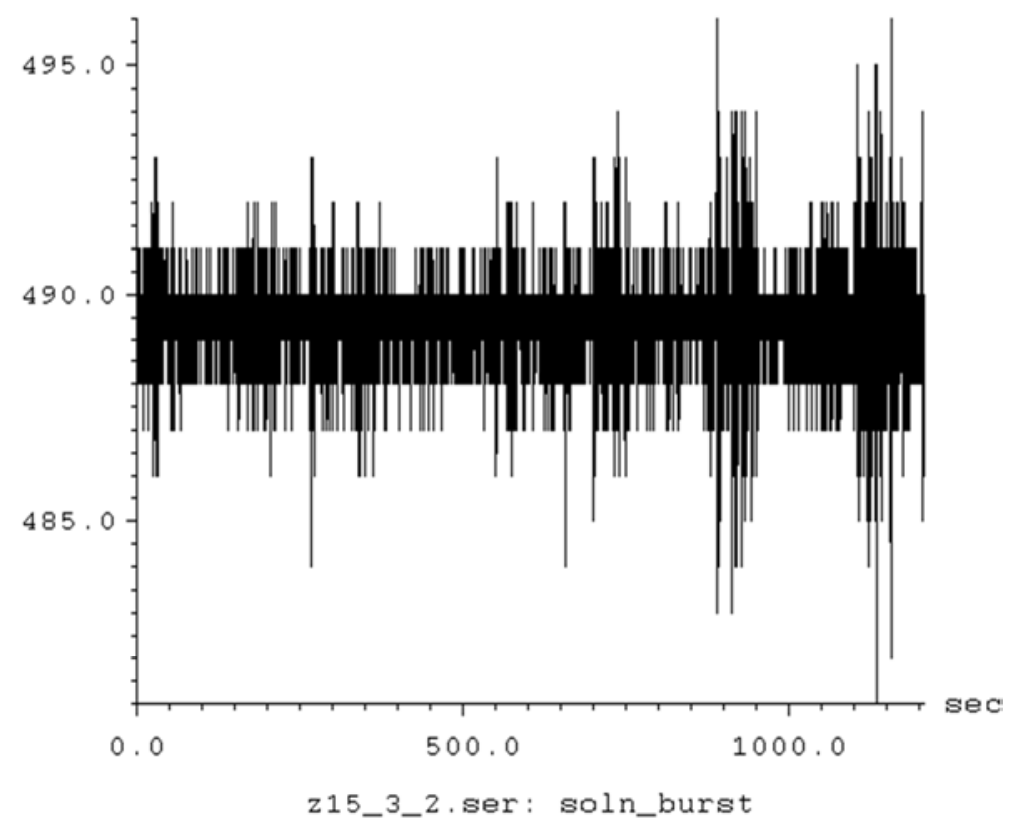

Figure 4. Eexample of a recording area of seismic noise recorded at the seismic Apollo 15.

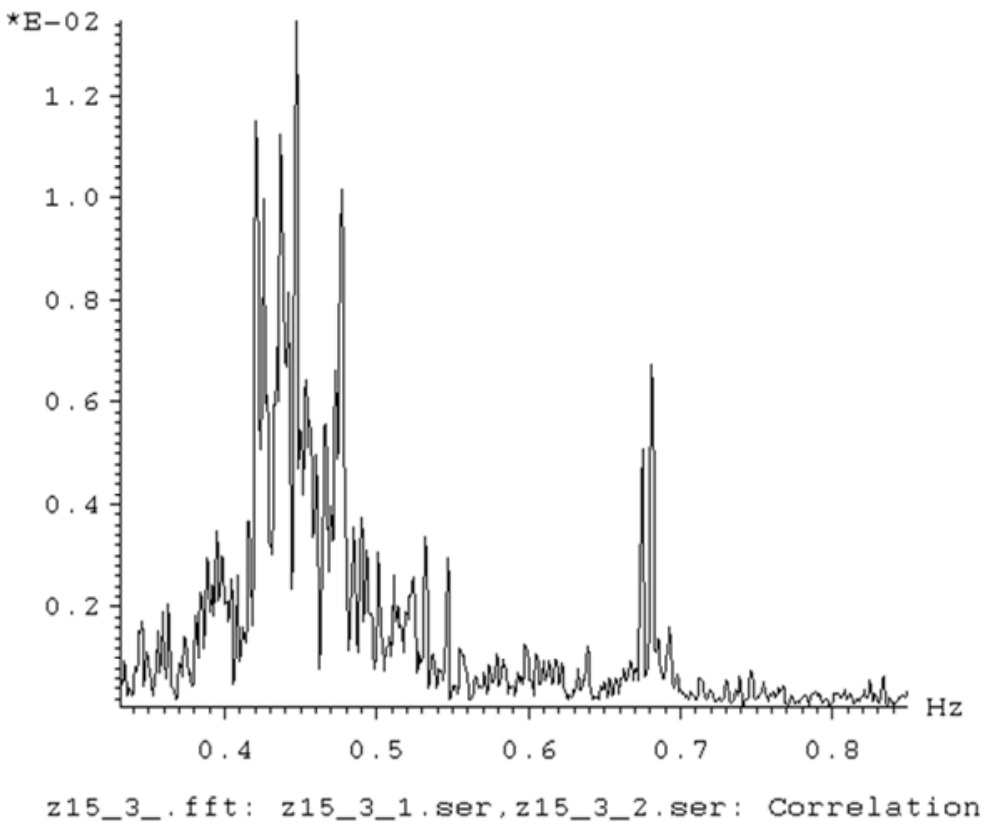

Figure 5. The energy spectrum of Figure 4 record.

Significant spectral peaks from the spectra of lunar Z-noise components, matching the frequency with sending pulses from the pulsar, as the seismic signals are compared with the data of [27] and tabulated (Table 1).

Table1. The comparison of the spectral peaks of seismic noise of the Moon frequency with the frequencies known pulsars [27].

\begin{tabular}{llll}
\hline № & Observed Spectrum Peaks (Hz) & Peaks [27] (Hz) & PSRJ \\
\hline 1 & 0.3492 & 0.34912 & $1803-1857$ \\
2 & 0.3557 & 0.34990 & $1831-1223$ \\
3 & 0.3589 & 0.35829 & $0746-4529$ \\
4 & 0.36297 & 0.36177 & $1322-5329$ \\
5 & 0.3737 & 0.375505 & 107 \\
6 & 0.3808 & & $1901+0413$ \\
7 & 0.3848 & 0.385055 & \\
8 & 0.3880 & & $0343-3000$ \\
9 & 0.3945 & & 71 \\
\end{tabular}




\begin{tabular}{|c|c|c|c|c|}
\hline № & Observed Spectrum Peaks (Hz) & Peaks [27] (Hz) & PSRJ & № PSRJ \\
\hline 10 & 0.3985 & 0.397149 & $1012-2337$ & 241 \\
\hline 11 & 0.4042 & 0.402608 & $1324-6302$ & 433 \\
\hline 12 & 0.4082 & 0.407396 & $1701-3766$ & 810 \\
\hline 13 & 0.4155 & 0.415347 & $1032-5206$ & 262 \\
\hline 14 & 0.4204 & & & \\
\hline 15 & 0.4252 & 0.425216 & $0157+6212$ & 55 \\
\hline 16 & 0.4365 & 0.438461 & $1119-7936$ & 310 \\
\hline 17 & 0.4414 & & & \\
\hline 18 & 0.4470 & & & \\
\hline 19 & 0.4535 & & & \\
\hline 20 & 0.4559 & 0.454065 & $1049-5833$ & 278 \\
\hline 21 & 0.4600 & 0.461748 & $1831-1329$ & 1260 \\
\hline 22 & 0.4665 & 0.468693 & $1012-5830$ & 244 \\
\hline 23 & 0.4729 & 0.472774 & $0055+5117$ & 33 \\
\hline 24 & 0.4777 & 0.477751 & $1826-1131$ & 1234 \\
\hline 25 & 0.4850 & 0.485834 & $1915+0752$ & 1582 \\
\hline 24 & 0.4899 & & & \\
\hline 27 & 0.4931 & & & \\
\hline 28 & 0.5012 & 0.500776 & $1944+1755$ & 1652 \\
\hline 29 & 0.5109 & 0.511023 & $1901-1740$ & 1483 \\
\hline 30 & 0.5230 & 0.523169 & $1839-1238$ & 1320 \\
\hline 31 & 0.5319 & 0.533043 & $1837-1243$ & 1304 \\
\hline 32 & 0.5464 & 0.546192 & $1901+1306$ & 1479 \\
\hline 33 & 0.5546 & 0.54417 & $1018-1642$ & 551 \\
\hline 34 & 0.5974 & 0.597873 & $1418-5945$ & 495 \\
\hline 35 & 0.6386 & 0.638732 & $1720-1633$ & 882 \\
\hline 36 & 0.6750 & 0.676259 & $1328-4921$ & 442 \\
\hline 37 & 0.6807 & 0.681746 & $0231+7026$ & 62 \\
\hline 38 & 0.6928 & 0.693313 & $0754+3231$ & 162 \\
\hline 39 & 0.7122 & & & \\
\hline 40 & 0.7230 & 0.723353 & $1239+2453$ & 382 \\
\hline
\end{tabular}

From an examination of Table 1 should be: observed frequencies match the Moon seismic peaks at frequencies of pulsars 3-4 second mark that exclusively unlikely. Because the distribution options of radioactive elements in the lunar geological structures are known [25], the analysis of seismic noise Moon on X, Y, Z components at frequencies of pulsars, we can figure out the relationship of the spatial location of radioactive elements and the structure of seismic noise at the frequencies of the pulsars. Previously, other researchers in 1970 in the Middle East through the records of the vertical component of MS in the band 1-2 Hz spectral analysis has been demonstrated significantly narrow peak at a frequency of $1,667 \mathrm{~Hz}$, corresponding to the pulsar CP 1133 [1]. Established its variations associated with characteristic points ephemeris, crossing the pulsar day horizon line, the upper and lower state. Further, one of the authors [1] on the moon in the Apollo seismic network records revealed the same pulsar CP 1133 . These seismic signals in accordance with the scientific paradigm of the time could be attributed to the gravitationalwave interaction of the Earth and the pulsar radiation. However, estimates of the power of the seismic signal by many orders of magnitude higher than estimated, that the "excluded" astrophysical mechanism of pulsar registered peak. Under pressure from the scientific community of the authors [1] gave up their results. No less scientific and administrative pressure and subjected to other researchers, reaching the footsteps of [1], for example, [2]. But the problem was just ignoring (or rather ignorance of the scientific community) powerful neutrino flux from the pulsar, and the effective interaction of the flow with the radioactive elements of geological structures [12-14]. There are based on direct seismic data from the Apollo 2.08. at 10.08. 1972. They were processed recording noise and variation spectra obtained SR1133 for all components of the dates indicated on seismic Apollon15. Figure $6 \mathrm{z}, \mathrm{x}, \mathrm{y}$, these spectra are presented.

Consider the spectral peak amplitude variations from the pulsar SR1133 using the absolute value of $\hat{\mathrm{S}}$. $\hat{\mathrm{S}}=(\mathrm{X} 2+\mathrm{Y} 2+$ Z2) 0,5. (Look Table 2).

Table 2. Variation of the absolute (S)amplitude of SR1133 pulsar and components of it from the 2.08 at 10.08 at 1972 A15.

\begin{tabular}{llllll}
\hline Data & $\mathbf{X}$ & $\mathbf{Y}$ & $\mathbf{Z}$ & $\hat{\mathbf{S}}$ & Note \\
\hline 2.08 .72 & 3061 & 2576 & 2405 & 4667.9 & \\
3.08 .72 & 6373 & 1445 & 1784 & 6773.9 & \\
4.08 .72 & 2596 & 1779 & 2333 & 3917.5 & \\
5.08 .72 & 1357 & 1728 & 3031 & 3743.6 & \\
6.08 .72 & 4172 & 2555 & 2681 & 5578.8 & \\
7.08 .72 & 2854 & 2243 & 3482 & 5030.0 & \\
8.08 .72 & 2665 & 1703 & 3066 & 4404.9 & \\
9.08 .72 & 2170 & 3124 & 2396 & 4495.4 & \\
10.08 .72 & 2449 & 2531 & 2666 & 4417.1 & \\
\hline
\end{tabular}

According to Figure 6 on $\mathrm{Z}$ component spectra in addition to the spectral component of the pulsar signals is virtually no other, while the horizontal components $\mathrm{X}, \mathrm{Y}$ (Figure $6 \mathrm{~b}, \mathrm{c}$ ) are observed interference of unknown nature. From Table 2, it follows - changing the polarization of the pulsar signal and the absolute value of the amplitude. The above features may be due to the surface shape of the source of the $\mathrm{X}$, Y waves, that is, the distribution of radioactive structures. Valid for Z- components of 
the main source of noise - wave response in the form of P waves on the neutrino flux from the pulsar located in the mantle of radioactive elements. The amplitude of $\mathrm{P}$ - waves dominate weakly susceptible to distortion. For surface X, Y - as the main source of the waves - wave reaction of radioactive elements, but located in the surface structures of the cortex. Surface waves in comparison with the $\mathrm{P}$ - waves - have a significant non-linearity, and interacts more easily transformed into noise, and therefore there are such differences (difficulties) in the allocation of spectrum peaks in the frequency pulsars. Figure 7 shows the Swann records Z- components pulsar SR1133 for 8,9,10 August 1972. Swann records X, Y- component for August 8.

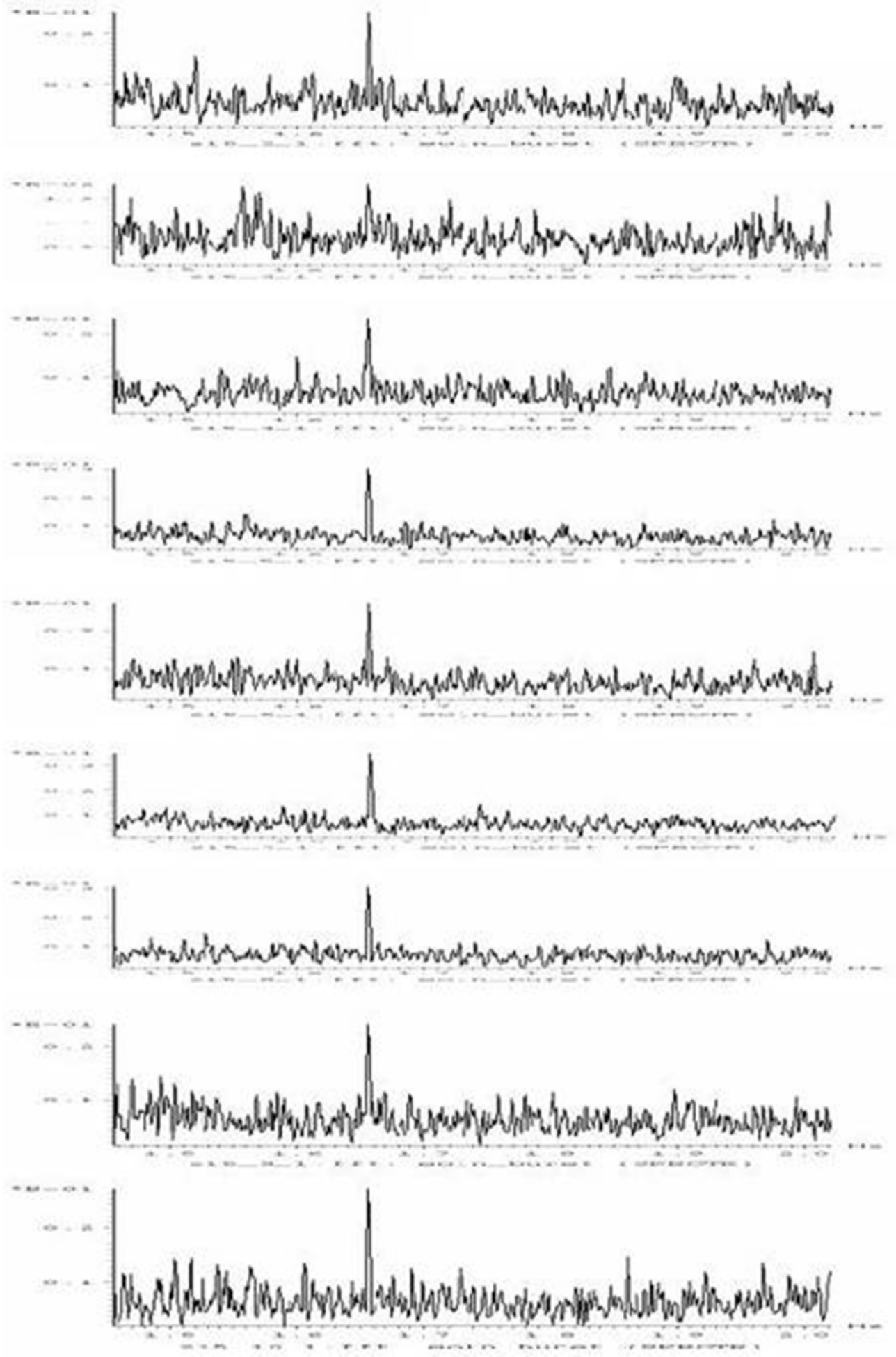

Figure 6. There are variations of the spectral peak from the pulsar SR1133 according to Z-component seismic noise from 2.08. to 10.08. 1972. 
International Journal of Astrophysics and Space Science 2017; 5(6): 85-99

91

X Y
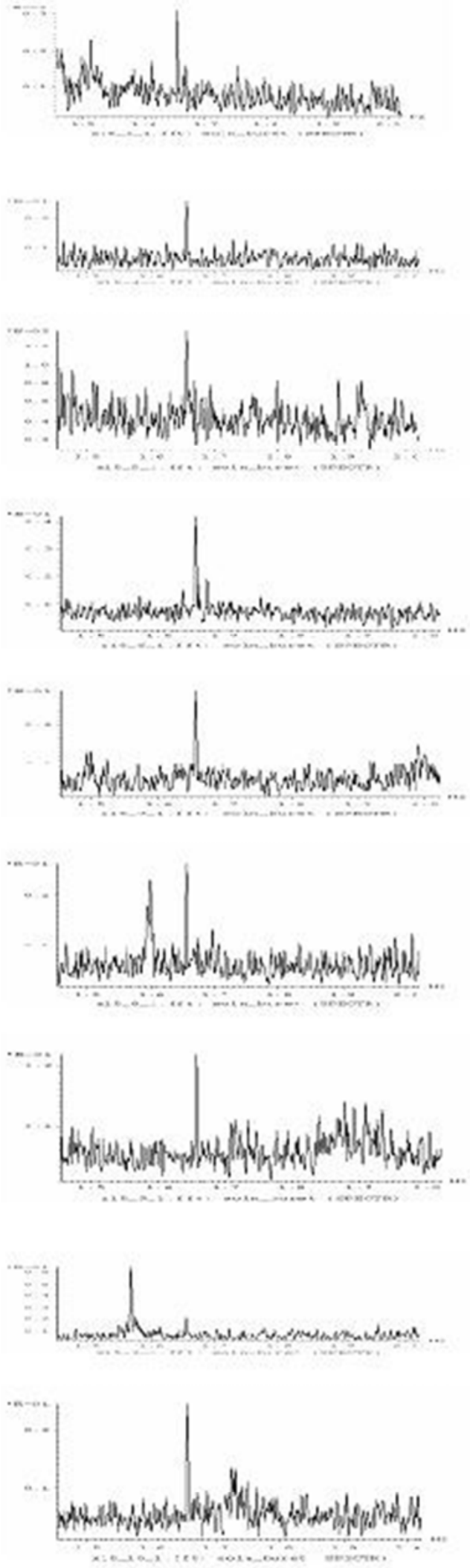
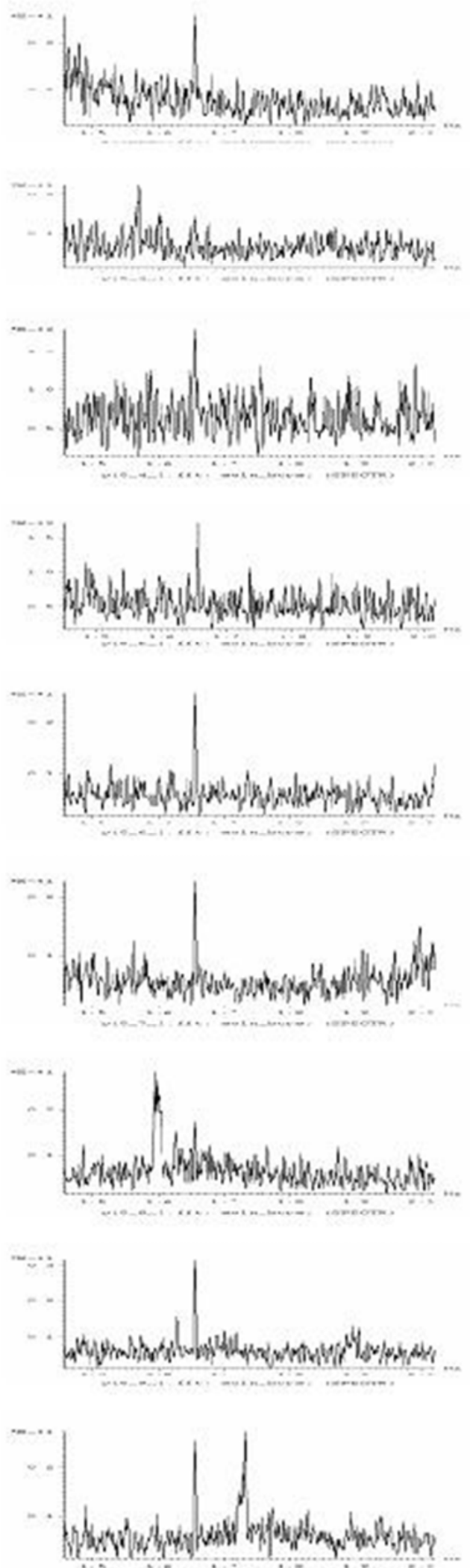

Figure 6. $b$ c Amplitude variations of the spectral pulsar SR 1133 ac peak according to X-component seismic noise (b) and the Y-component (B) to 2.08. at 10.08. $1972 y$. 


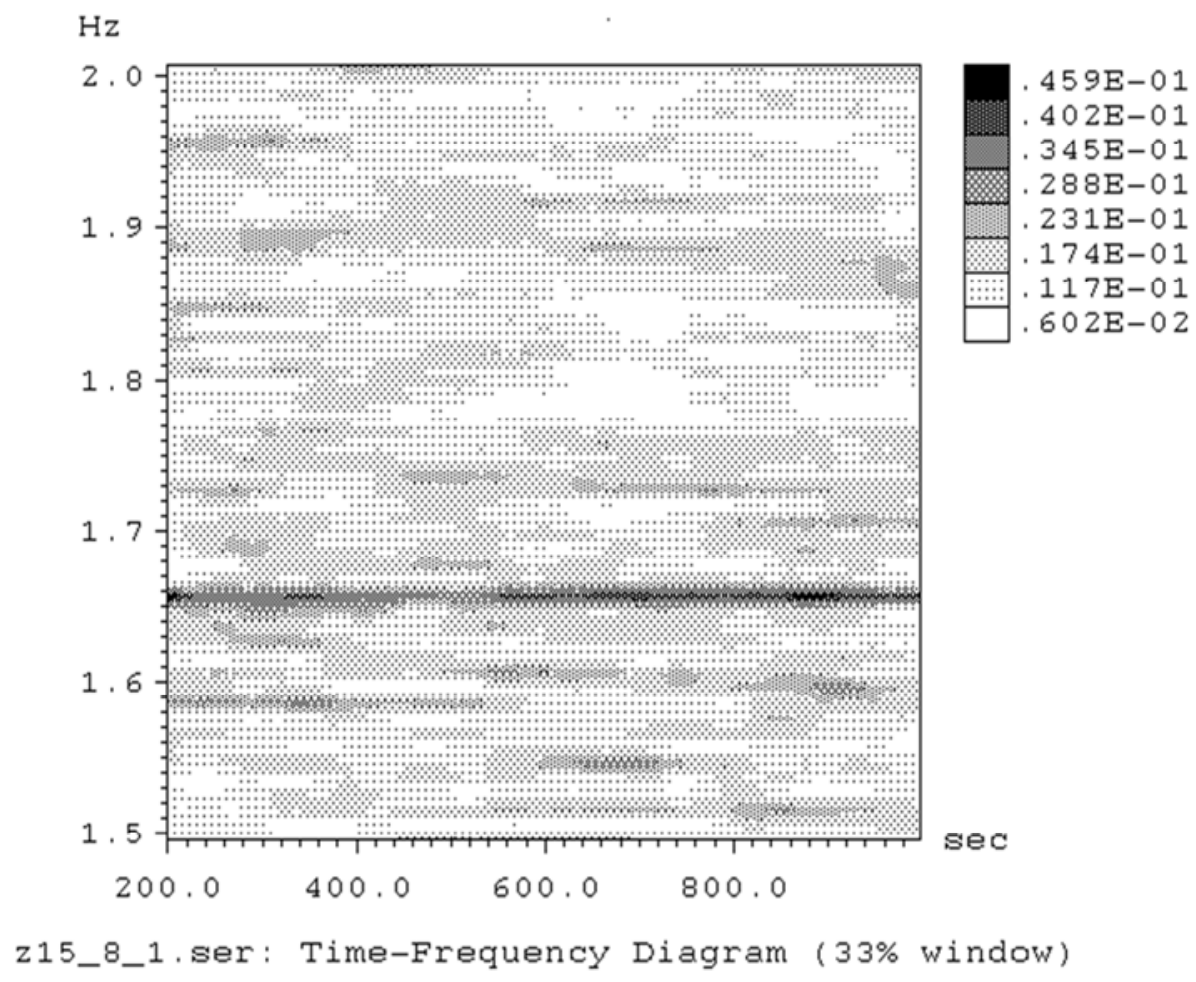

A

Figure 7A. STAN recording Z- components pulsar SR1133 for August 8, 1972.

In addition to the main signal at a frequency of $1,667 \mathrm{~Hz}$ STAN clearly visible perturbations at frequencies of $1.59 ; 1,55$; $1.73 ; 1,89 ; 1,93 ; 1,96 \mathrm{Gts}$.

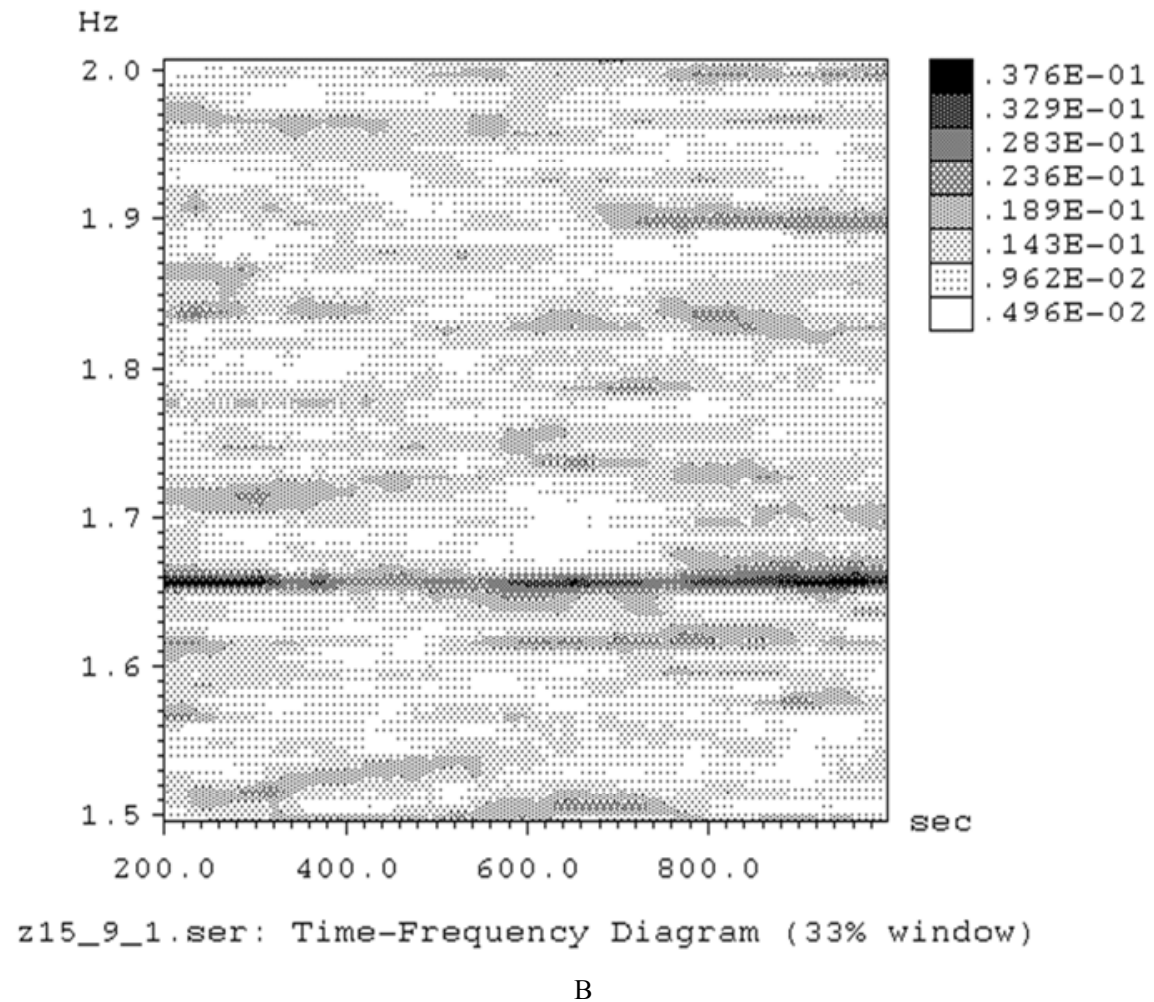

Figure 7B. SWAN (STAN) -Spectrum Timing Analyzing of record Z- components pulsar SR1133 for August 9, 1972.

As in Swan And also noticeable perturbations at frequencies of 1.51; 1,57-1,58; 1,6-1,62;1,173-1,71-1,72;1.82; 19; 1.97; $2.0 \mathrm{~Hz}$. 


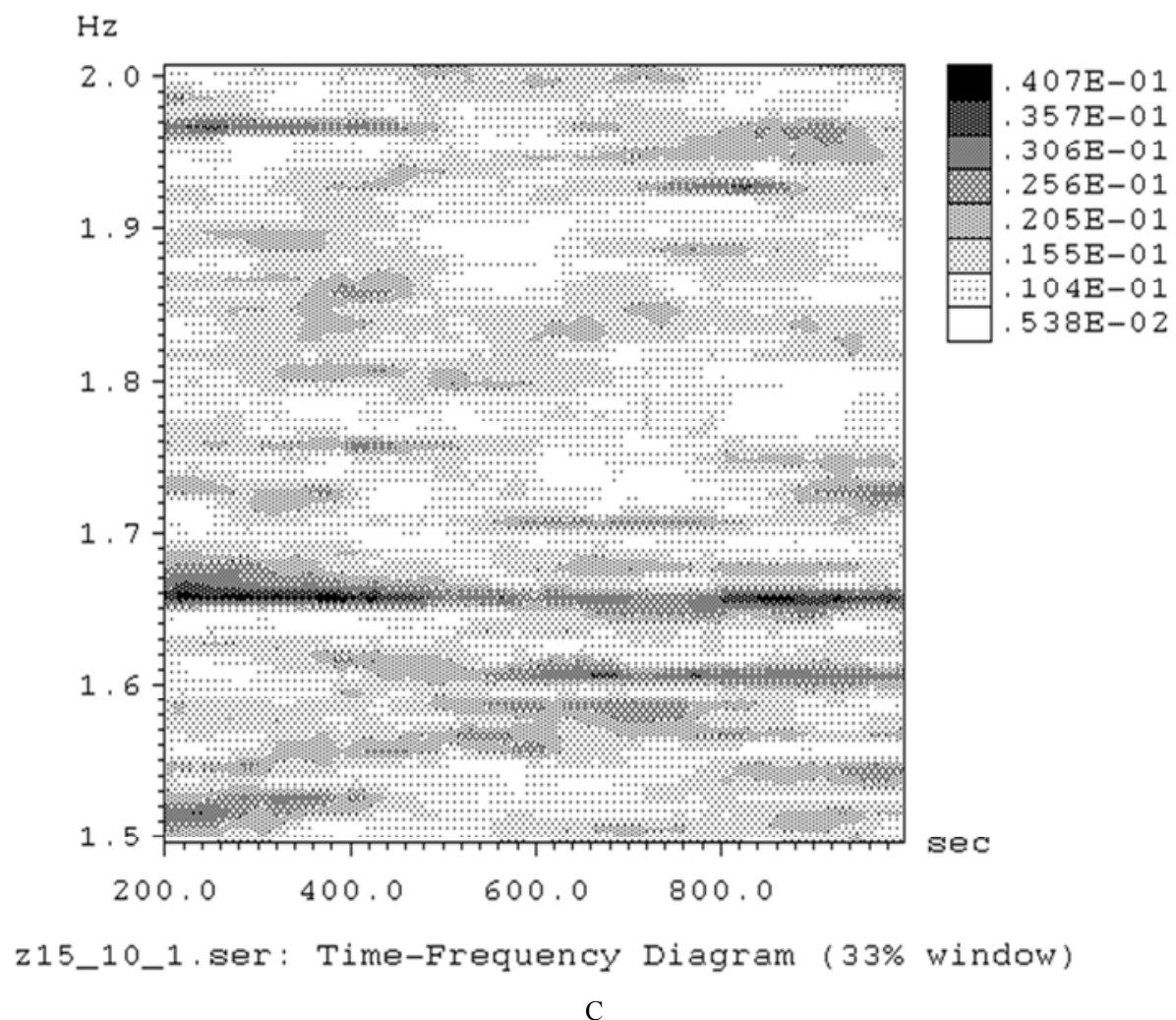

Figure 7C. STAN Z-component for August 10, 1972.

As in previous sections of Swan has entries at $1,52-1,54 ; 1.551 .58 ; 1,62-1,61 ; 1.72-1,71-1,73 ; 1,75-1,73 ; 1,89 ; 193-$ $1.97 \mathrm{~Hz}$.

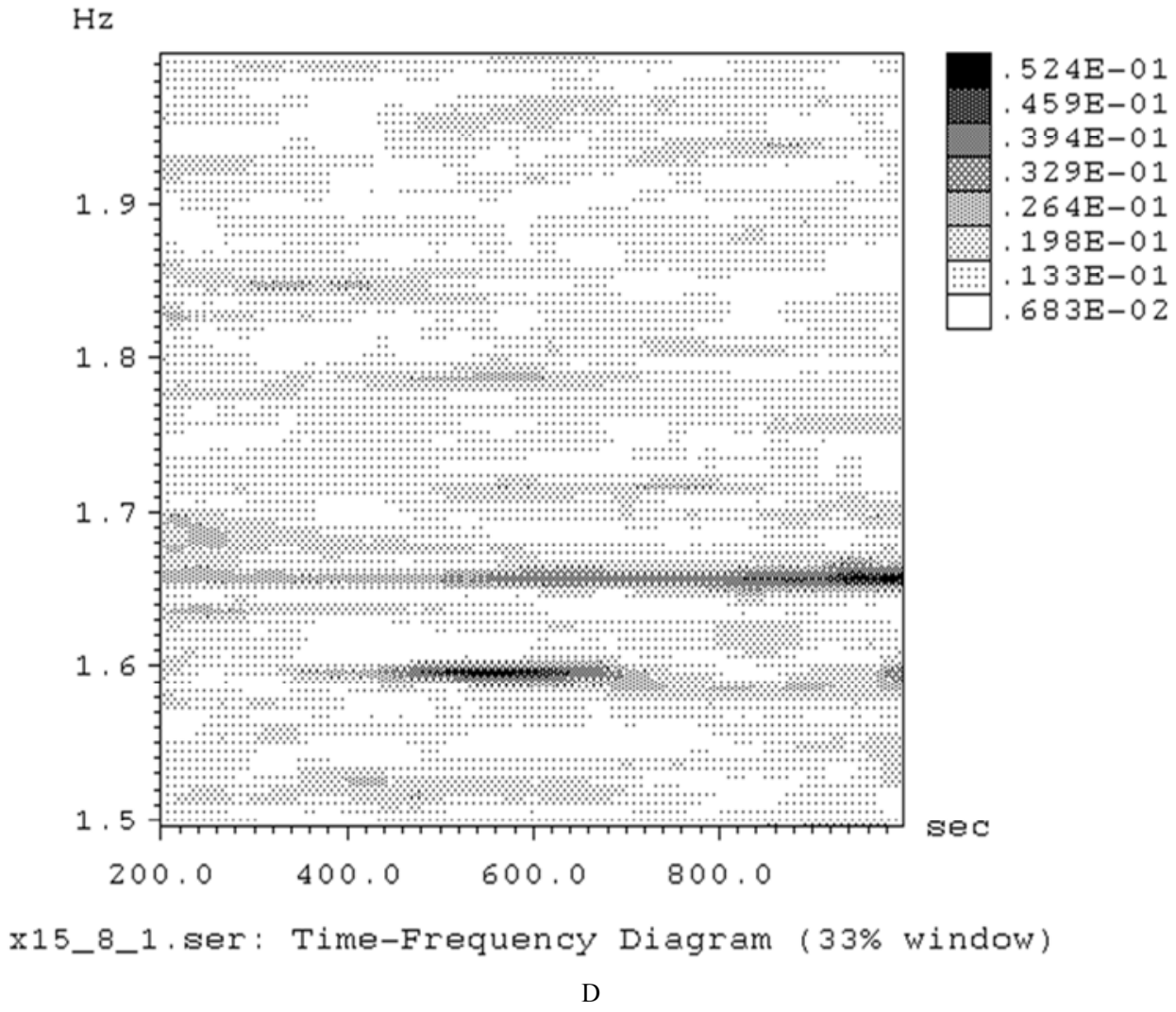

Figure 7D. STAN recording of X-component of pulsar SR1133 for August 8, 1972. 
This Swan seeing strong weakening of the main signal at a frequency of $1,667 \mathrm{~Hz}$ at the initial site entry clearly visible only at a frequency of disturbance $1,59 \mathrm{~Hz}$; faintly visible signal on 1,$85 ; 1,72 \mathrm{~Hz}$.

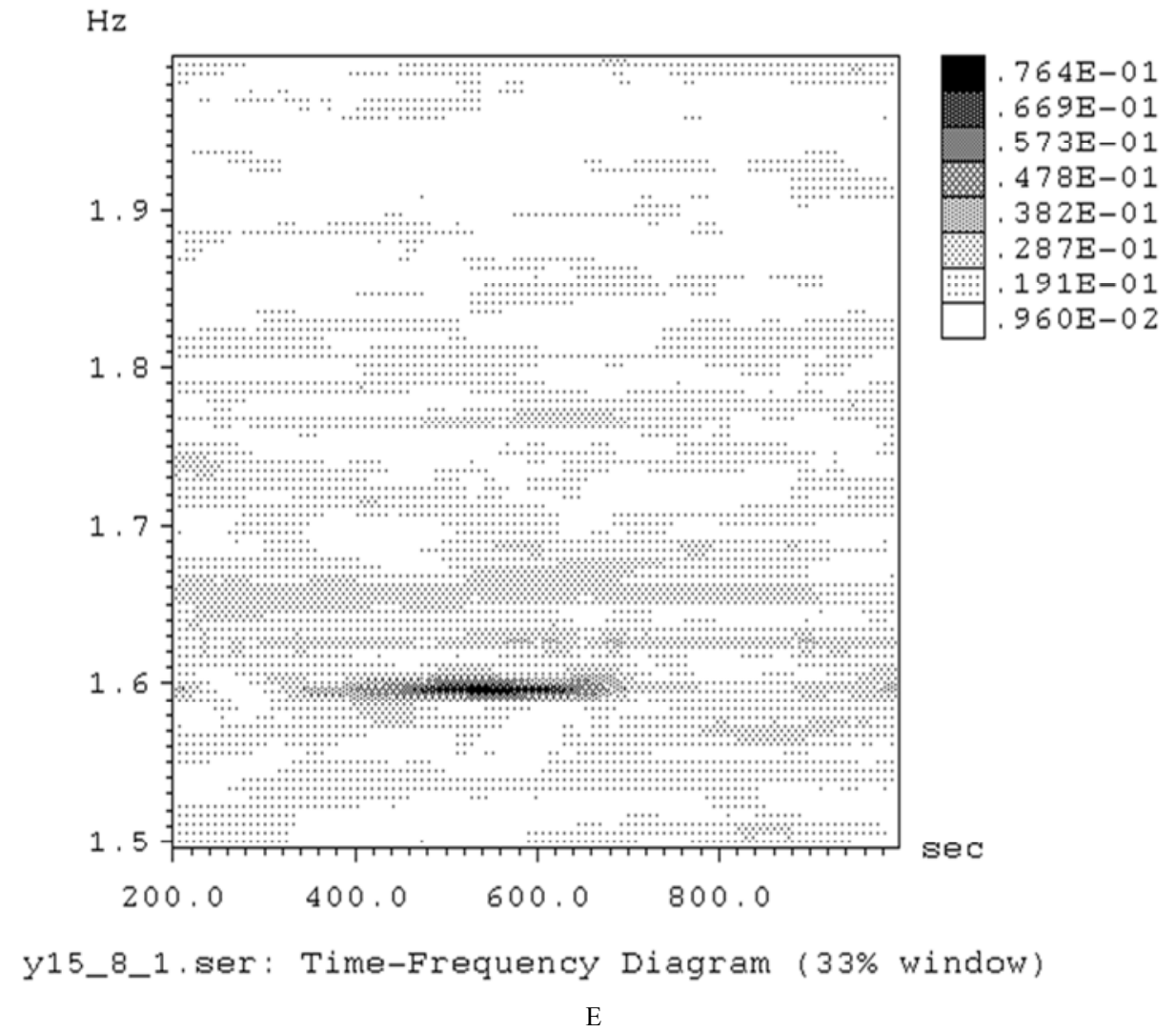

Figure 7E. STAN recording of Y-components pulsar SR1133 for August 8, 1972.

In Swan record Y- components existence signal from the pulsar SR1133 very ghostly, there is only a signal at a frequency $1,61 \mathrm{~Hz}$. Each component (Swan Z-component, Figure 7 A, B, C) contains the real high frequency components which determine the nature of the ciliated Swann pulsar SR1133. Moreover, these components are also effects of neutrino fluxes from other pulsars [26]. They are easy to find in the Catalog, for example, J0045-7042 1,5814Gts; J0206-4028 1,5859Gts; J0417 + 35 1,5281Gts; J0502 + 4654 1,556Gts; J0729-1836 1,96Gts; J0215 + 6218 1,8218Gts; J0401-7608 1,834Gts; J0452$17591,821 \mathrm{Gts} ; \mathrm{J} 0532-6639-1,555 \mathrm{~Hz}$. By comparing the frequency found on Swan, note, in addition to the fundamental frequency $1,667 \mathrm{~Hz}$ observed in the form of segments of varying duration plots at frequencies of pulsars Catalog [26]. In general, all three STAN demonstrate one of the effects of nonlinear seismology that is synchronization and decay instability of quasi-harmonic seismic signal [23]. SWAN of X- component (Figure 7, E) contains signals at $1.59 ; 1.72 ; 1,85 \mathrm{~Hz}$. That is observed in the $\mathrm{Z}$ - component. SWAN of Y- component contains only one signal at a frequency of $1,61 \mathrm{~Hz}$ corresponding pulsar J1058- 5957. It should be emphasized - the primary signal (or rather its education) as stable as the speed of the pulsar, and all other changes in the seismic signal as the response of radioactive rocks on the Moon the impact of the neutrino flux from the pulsar is due to the nonlinearity of the seismic wave field. It is possible that this mechanism is a considerable degree generates the frequency composition of the lunar MS (microseisms) or noise. Another question arises - how to explain the significant temporal variation of the amplitudes of the useful signals and even the disappearance of many of them briefly or permanently, depending on the coordinates of the registration. By the nature of the radiation parameters of the neutrino flux from the pulsar must be sufficiently stable and amplitude (as is observed for other types of radiation), and seismic nonlinear effects are easily distinguishable, and usually are not so great. Abandon another important parameter of the interaction of neutrinos and radioactive rocks of the Moon - the radiation pattern. Consider the simple case of an antenna from a rectangular slab of thickness $\mathrm{d}$ with sides $\mathrm{A}, \mathrm{B}, \mathrm{A}>\mathrm{B}$. The radiation response of the plate on the neutrino flux is determined by the percentage of radioactive nuclei, the energy characteristic of the neutrino flux and the total number of neutrinos passing through the volume of $\mathrm{A} \times \mathrm{B} \times \mathrm{d}$. The response of the antenna at a flow normal to the surface of A x B and the normal to the surface of the ribs Bxd, ceteris paribus characterized as A / d, with $\mathrm{A} \gg \mathrm{d}$, that is we have the case of the dipole gain antenna. This agrees well with the data on the signal amplitude Figure 7 , where it plays an important role as superficial deposits. For deep compact sphere-like deposits of the radiation pattern is 
also close to the area that is well observed in the Zcomponent. The structure of the surface deposits of properties displayed signals $\mathrm{X}, \mathrm{Y}$ - component of their disappearance and / or attenuation. The proposed model is based on the normal direction of flow of particles in the case

\section{Seismic signals}
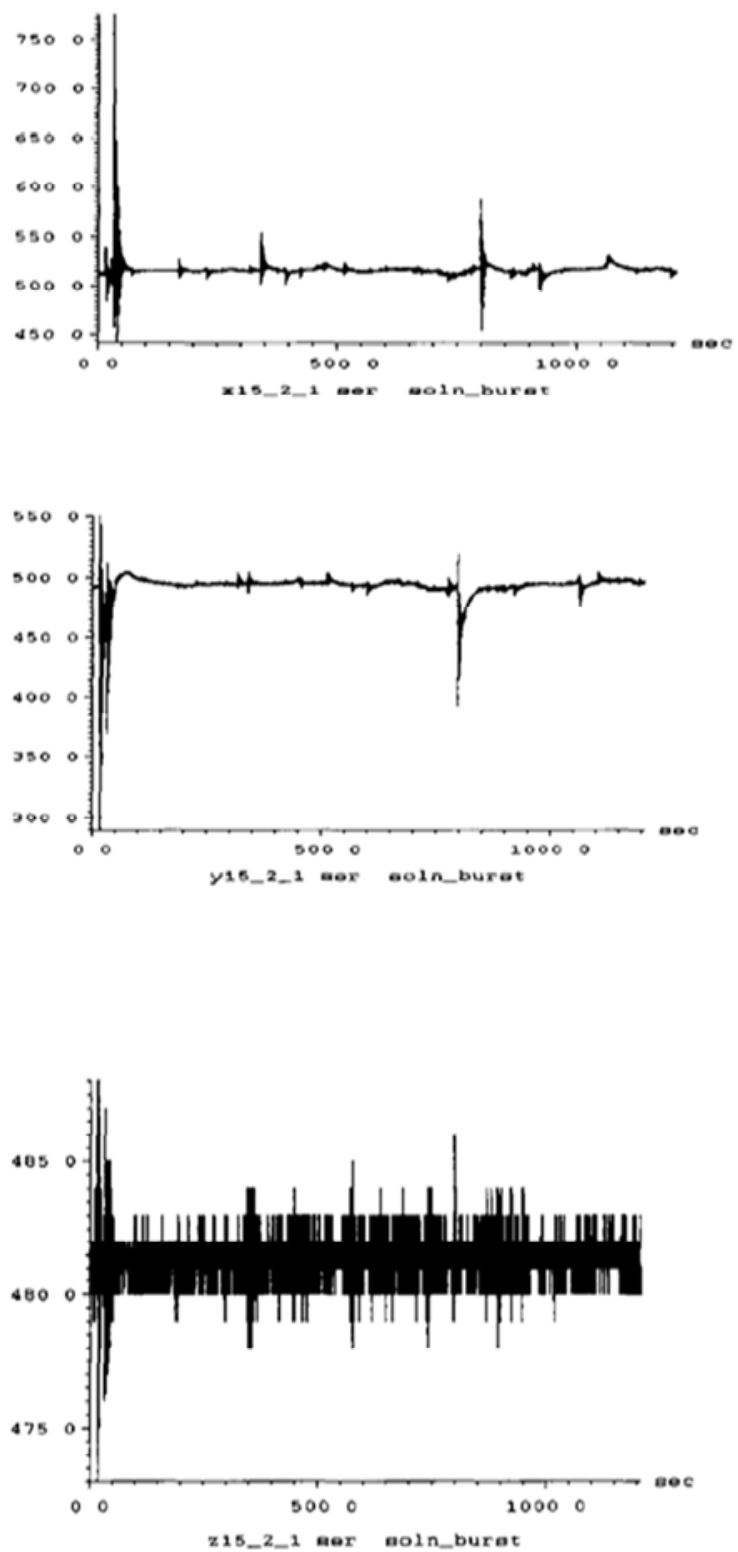

of existence of quantum particles in the flow of synchronicity focus is likely to be presented in a different form.

Other approaches to the study of the structure of the lunar signals are shown in Figure 8.

The spectrum signals
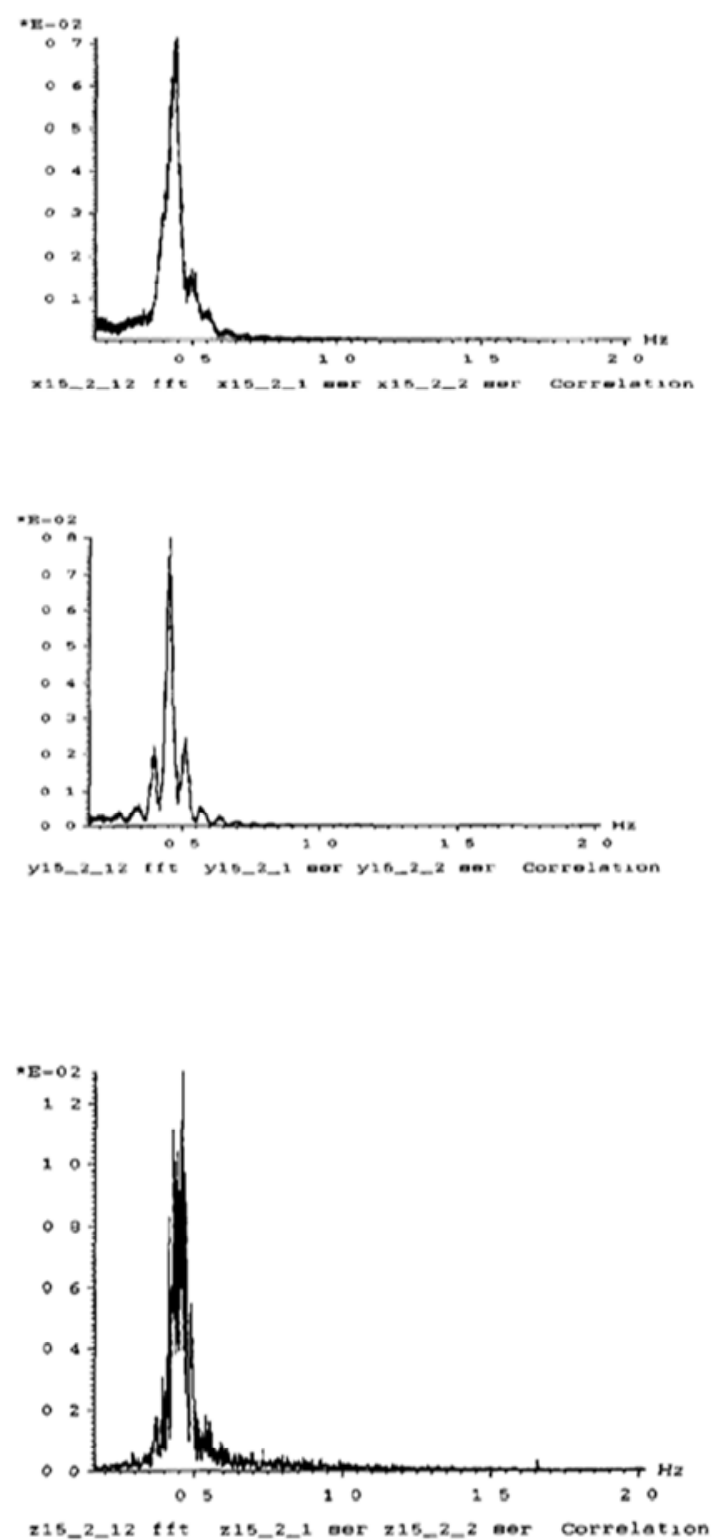

Figure 8. The record of seismic signals received at seismic network and the Apollo spectra.

Background micro cracks and/or seismic acoustic noise of the lunar crust in time processing data smoothed out the shape of the spectra (Figure 8). In Table 1, not all of the narrow spectral peaks coincide with the respective frequencies of pulsars; probably account and found seismic impact on the moon at frequencies unknown pulsars, are not included in the catalog [26] or signals at these frequencies the result of the seismic wave field of non-linearity of the moon's crust (1st harmonic, sub harmonic, decay instability).
4. Search and Investigation of the Occurrence of Multiple (Double) Star Systems in Seismic Fields of the Moon, and Comparison with Other Variations of Terrestrial Processes

Under other processes or analysis should first take decay of radon and radioactive ore in the world Baikal. Both 
processes are influenced neutrino fluxes, especially solar. radon trans Baikal ore by averaging the hourly interval Figure 910 presents fragments of variations of radiation and duration $1351 \mathrm{~h} . ., 56.3 \mathrm{~d}$.

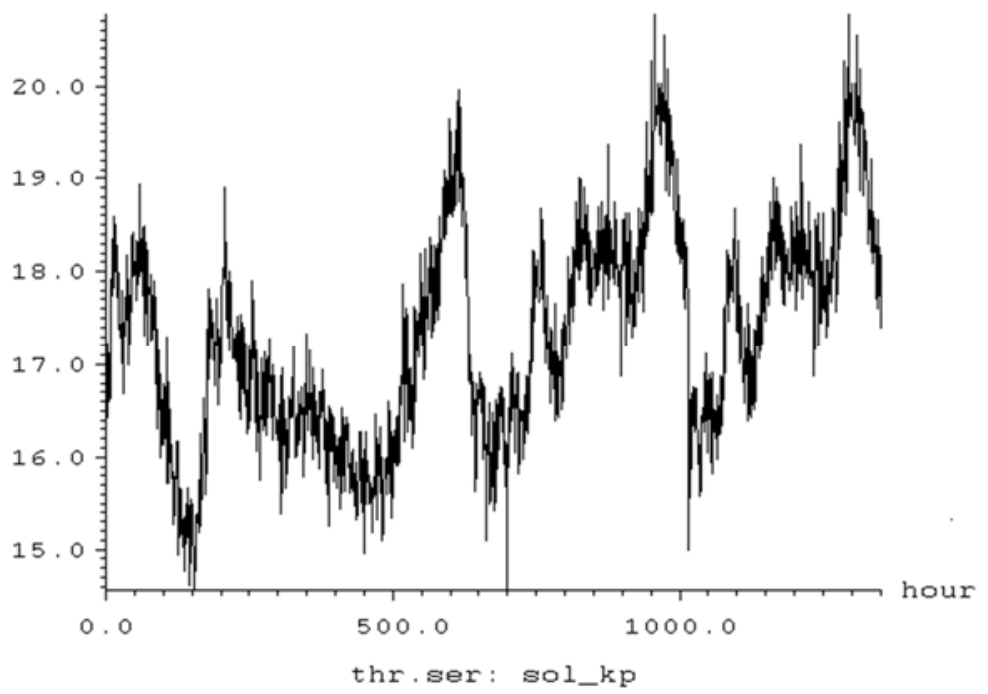

Figure 9. Detail of simultaneous recording with variations in radiation levels of radon.

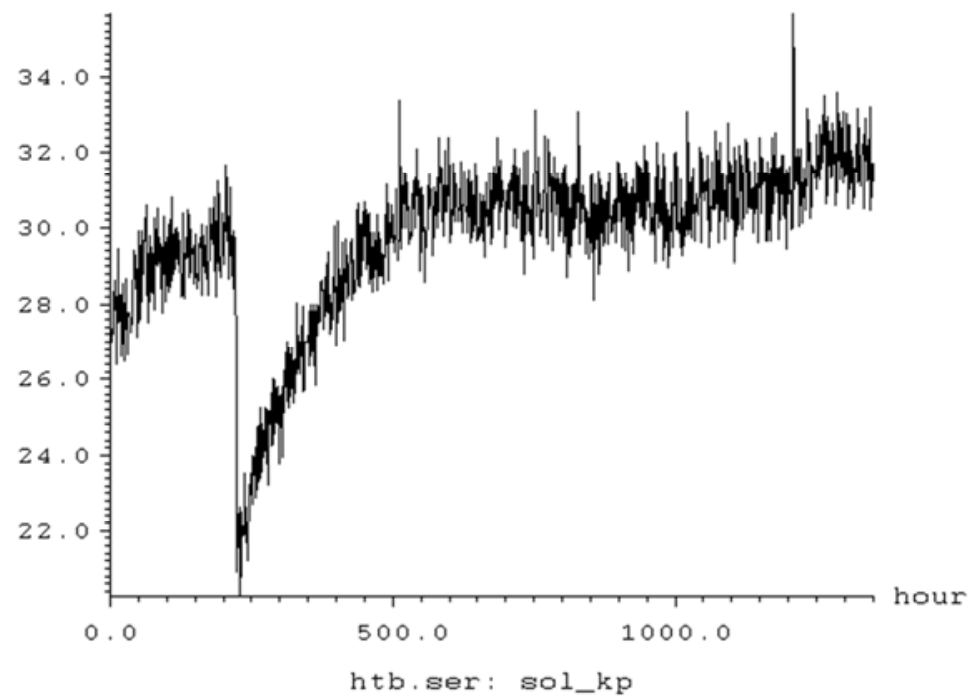

Figure 10. Fragment simultaneous recording with variations in radiation levels transbaikal ore.

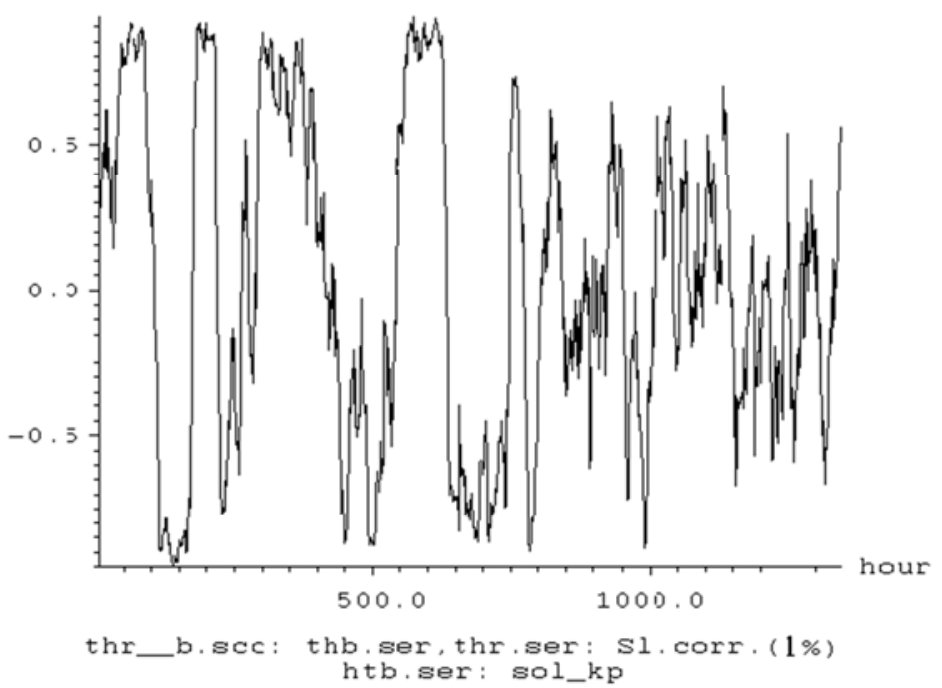

Figure 11. Sliding correlation between variations in the intensity of radon and Baikal ore. 
As a variation of radon radiation levels (Figure 9), and a sliding correlation between variations in the intensity of radon and Baikal ores (Figure 11) contain the disturbance, especially for radon. Smooth enough radiation levels ore contrasts with the level of radon. Variations in ore sufficiently studied and do not have significant emissions $[12,13]$. Considerable scope disturbances radon can partly be explained seismic geological processes, but can not be ignored is another difference that was manifested in time characteristics of signals from the pulsar in the Moon - their dependence on the shape of the radioactive mass (or antenna). Radioactive ore - a point receiver, radon field extended and orientation of these antennas is different. There is a high significant correlation between the variations and the intensity of the anti-correlation of radon and Baikal ore, both positive and negative very large intervals (up to several days). Moreover, if the jump in variations of ore was due to technical problems related to the location of ore into a separate container and the subsequent reduction of its intensity from 22 to 28-30un. That has got to peak in anti variations of radon.

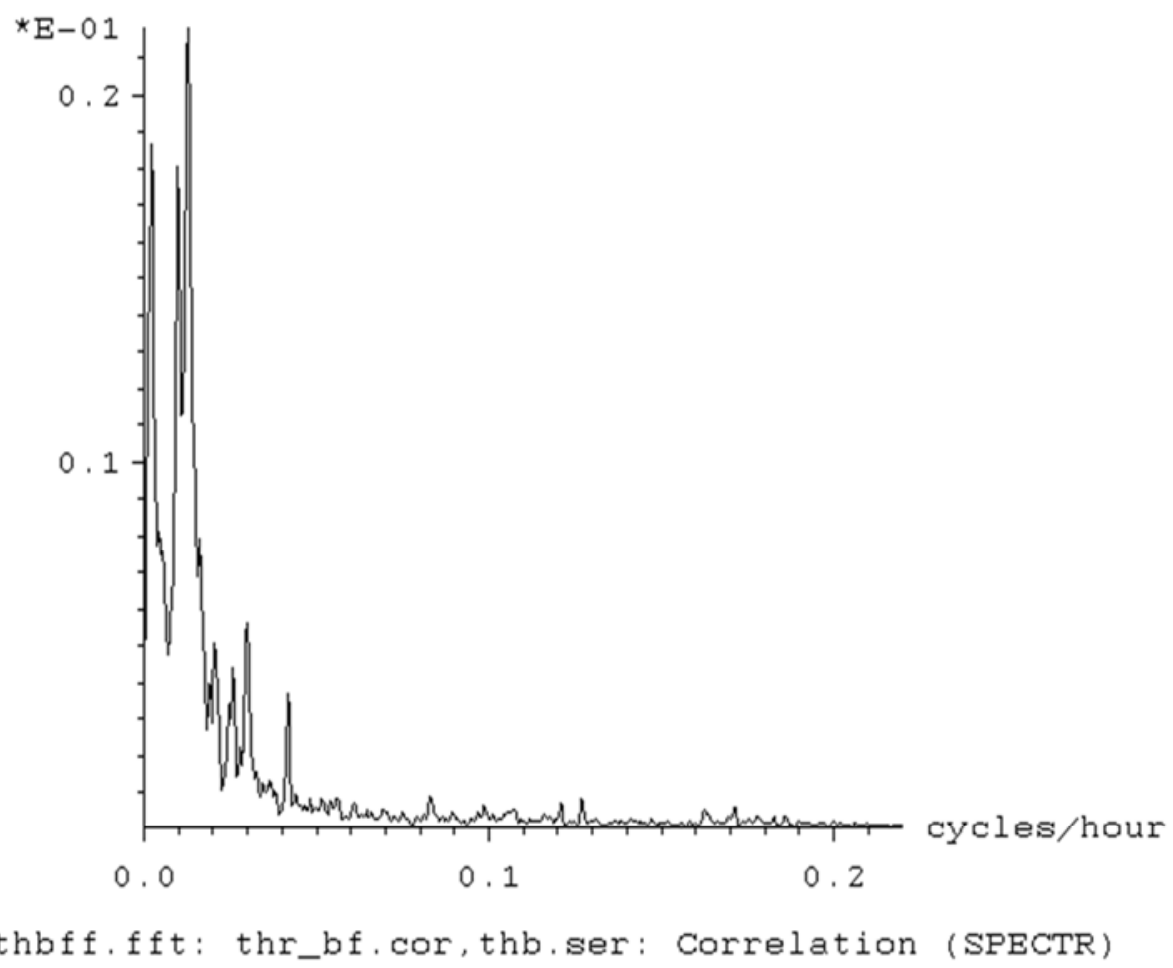

Figure 12. The spectrum of variations in the intensity of radiation Baikal ore.

In all probability distribution of radon in the earth's crust resembles a set of plate antennas. According to K. Spiring addition pulsars promising astrophysical sources modulated neutrino flux are close multiple (usually dual) system [10]. The flow rate is highly dependent on certain parameters such as the energy spectrum of gamma -radiation and relationships ${ }_{v} / \gamma$, due to the thickness of material around the source. Point sources of high-energy neutrinos - binary systems of neutron stars, sources of gamma - radiation in the $\mathrm{TeV}$ - rated range of radiation. There is another feature - the interaction of neutrinos with the Earth's ionosphere (Table3).

Significant periods of sliding correlation spectra variations of uranium ore and radon variations and periods of seismicity of the Moon, the closest binary systems and the natural vibrations of the sun.

Table 3. Significant periods of sliding correlation spectra variations of uranium ore and radon variations and periods of seismicity of the Moon, the closest binary systems and the natural vibrations of the Sun.

\begin{tabular}{|c|c|c|c|c|c|c|c|}
\hline № & I & II & III & IV & V & VI & Note \\
\hline 1 & $28.44 d$ & $28.4 d$ & $27.5 \mathrm{~d}$ & & & & Об. Сл./Пр. Л \\
\hline 2 & $10.66 \mathrm{~d}$ & $21.3 \mathrm{~d}$ & $13.8 \mathrm{~d}$ & & & & Lunar tide \\
\hline 3 & 7.76 & $8.53 \mathrm{~d}$ & $6.7 \mathrm{~d}$ & $6.21 \backslash 6.89 \mathrm{~d}$ & $1 \mathrm{kp}$ & & CV Vel \\
\hline 4 & $5.64 \mathrm{~d}$ & $6.10 \mathrm{~d}$ & & & & & \\
\hline 5 & $4.49 \mathrm{~d}$ & $5.02 \mathrm{~d}$ & & $4.45 \mathrm{~d}$ & $3 \mathrm{kp}$ & & V356Sgr \\
\hline 6 & $3.88 \mathrm{~d}$ & $3.41 \mathrm{~d}$ & $3.5 \mathrm{~d}$ & $3.44 / 3.58 \mathrm{~d}$ & $1 \mathrm{kp}, 0.1$ & & CVVel, hAqv, \\
\hline 7 & $2.19 \mathrm{~d}$ & & $2.25 \mathrm{~d}$ & $2.20 \mathrm{~d}$ & $9 \mathrm{kp}$ & & UwCma \\
\hline 8 & & & $2.03 \mathrm{~d}$ & $2.03 \mathrm{~d}$ & $0.7 \mathrm{kp}$ & & AG Per \\
\hline 9 & $1.33 \mathrm{~d}$ & & $1.33 \mathrm{~d}$ & $1.33 \mathrm{~d}$ & $0.25 \mathrm{kp}$ & & V906 Sco \\
\hline
\end{tabular}




\begin{tabular}{|c|c|c|c|c|c|c|c|}
\hline № & I & II & III & IV & V & VI & Note \\
\hline 10 & $23.3 \mathrm{~h}$ & $24.09 \mathrm{~h}$ & $23.3 \mathrm{~h}$ & $23.4 \mathrm{~h}$ & $0.5 \mathrm{kp}$ & & GAqv \\
\hline 11 & $11.4 \mathrm{~h}$ & $12.04 \mathrm{~h}$ & & & & & \\
\hline 12 & 9.94h & & $9.90 \mathrm{~h}$ & & & & \\
\hline 13 & $8.4 \mathrm{~h}$ & & $8.37 \mathrm{~h}$ & $8.232 \mathrm{~h}$ & $0.5 \mathrm{kp}$ & & VV U. Ma \\
\hline 14 & & $6.80 \mathrm{~h}$ & $7.76 \mathrm{~h}$ & $7.74 \mathrm{~h}$ & $0.04 \mathrm{kp}$ & & YY Eri \\
\hline 15 & & $6.17 \mathrm{~h}$ & $6.4 \mathrm{~h}$ & $6.42 \mathrm{~h}$ & $0.012 \mathrm{kp}$ & & i Boo \\
\hline 16 & & $4.05 \mathrm{~h}$ & $3.85 \mathrm{~h}$ & $3.85 \mathrm{~h}$ & $0.074 \mathrm{kp}$ & & SW Lac \\
\hline 17 & & & $3.42 \mathrm{~h}$ & $3.43 \mathrm{~h}$ & $0.1 \mathrm{kp}$ & & j. U. Min \\
\hline 18 & & $2.995 \mathrm{~h}$ & $3.03 \mathrm{~h}$ & & & & \\
\hline 19 & & $179 \min$ & & & & $175.9 \min$ & g16, 13 \\
\hline 20 & & $160.4 \mathrm{~min}$ & & & & $158,9 \mathrm{~min}$ & $\mathrm{~g} 10,12$ \\
\hline 21 & & 156.7 & & & & $156,5 \mathrm{~min}$ & $\mathrm{~g} 14,13$ \\
\hline
\end{tabular}

I -is the period moving correlation (day, hour), II - period variations of Baikal uranium ore (d, h), III - period variations of the Moon (d, h), IV - periods closest binaries ( $\mathrm{c} \mathrm{h}$ ), V - the distance to the dual system ( $\mathrm{kpc})$, VI - the natural oscillations of the Sun (min).

This follows from the fact that the observations of the Sun oscillation periods of instability in the temporal variations of the hydrogen maser [27]. If you look at the upper part of the Earth's ionosphere with a high content of hydrogen, it becomes obvious analogy with the plasma of the hydrogen maser as the corona of the sun, as well as other neutrino heating mechanism of the crown, which as in the galaxy may arise plasma zone of the coherent state. Therefore, the frequency of neutrino fluxes of multiple systems should be displayed in the fluctuations of radioactive structures on the Moon (see Table 3), and in others, such as plasma environments. Really study the Earth's ionosphere also led to the registration of hidden periodicities, coinciding precisely with the parameters of multiple systems, which is a great merit of prof. L. V. Grunskaya with the staff of the Vladimir University [28]. They registered the main components at frequencies of double star systems: J $0700+6418 ; 1012$; $1537+1155 ; 1959+2048 ; 2130+1210 ; 1915+1606 ;$ c up to six significant figures.

But the most powerful neutrino flux density and possible energy occurs when a supernova explosion. [10] Perhaps the energy component is high enough, that should cause an increased level of seismicity of the Earth, which was first pointed out by Czech researchers found in a centuries-old statistics correlation between the outbreaks and catastrophic earthquakes. The important role of the neutrino energy specified in [29], there is also noted on the nature of the neutrino beam trigger some geophysical processes (earthquakes). Unfortunately, this only work with the model of the neutrino beam, the other - using interesting observational data have been completely closed in the model of gravitational radiation hopeless. Therefore the revision of existing database as a result of exposure to the Earth neutrino flux from the supernova explosion in the Large Magellan Cloud (SN 1987A) registered in the 10 to 40 UT on February 23 1987. By this time there was a kind of global network focused on the detection of gravitational spike (Italy), or stream of neutrinos - Japan, USSR (seismic events is not affected). In all the "classic" registration systems, for example, [30, 31] signals were observed, however, when interpreting any conflict, including at the time of registration, therefore, it was asked to geophysical data [32], as noted previously [29]. All this did not allow the cram signals were observed in the model of the gravitational effects, the classical idea of the interaction of the neutrino flux with existing reception also made unacceptable registered signals and the possibility of effective influence of the neutrino flux in the radioactive elements and the structure still waiting in the wings [12-15]. Note the properties of the seismic field of the planet (Earth) as a carrier of information and the interaction of the neutrino with radioactive geological structures, and probably with the electromagnetic processes in the ionosphere and the earth's core. $\mathrm{V}$ first micro seismic field of geological structures, enriched with radioactive elements and containing the impact of the seismic signals neutrino fluxes, will have the same spectral - time components that neutrino flux (see., eg, [12, 13]). The amplitude variations of these signals will also consist of the total pattern of radioactive elements structures, which is particularly evident when looking at the signals supernova SN 1987A. One consequence of this - the lack of coincidence in time recorded seismic signals and hardware [30 - 33]. Of great interest and signals defined probably top of the ionosphere, the pole areas of the Earth and simply electrical signals received by the coil magnet. In view of the above, with very high probability all seismic emissions [32] The origin of the flare. Consider the seismic data [33], which proposed to consider the Earth as a single Helios geophysics factors - in other words at the moment as the astrophysical detection, including neutrinos [12-15, 10, 27-28]. However, studies [33] guided by the gravitational waves from a supernova, that is very weak and the pulse signal, the capture cross section is $\sim 10^{-40}$, making the search for signals unpromising. Considering that for certain systems and radioactive geological structures capture cross section is appreciable $[12,24]$, interpret the findings and the results of [33] in the neutrino "key". For example in Figure 2 [33], the correlation coefficient between the events in the neutrino detector Mont - Blanc and the energy state of gravitational antennas of Rome and of Maryland takes the maximum value of accurate over time coincides with the Earth's seismic activity. The spectra of the temporal variations of the correlation coefficient contains significant peaks at 240, 185; 145 (145.9); 120 (120.2); 96 (95.38); 94 (95.38); 67.5 
(67.76); 53.5 (54.88); 43.5 (44.18); 40; (at 2 spectra simultaneously) (39, 53); 34.6 (32.19); 32 (32.19) minutes. The values of these spectral peaks (Figure 2 [33]) are in good agreement $(\sim 1-3 \%)$ with values periods of natural oscillations of the Sun (in brackets), which are modulated by the solar neutrino flux $[12,13]$. The spectrum of temporal variations of the correlation coefficient (Figure 3 [33]) according to the total energy states antennas Rome and Maryland also have significant peaks at 450;270; 170 (171.1); and 85 (84.4) minutes, some of which are also in good agreement with the periods of solar oscillations. However, there are more than 450 long-period oscillations; $270 ; 240 ; 185$ minutes, far exceeding all others, and that the data [34] are not available. They are not difficult to imagine how these sub harmonics solar periodicity period of 149.2 minutes, which has the third sub harmonic - 450min; period of 133.3 minutes with the 2 nd sub-harmonic of 270 minutes; 120.2 minutes with the 2 nd sub-harmonic of 240 minutes; 91.8 minutes in the second sub-harmonic $185 \mathrm{~min}$. The appearance of sub harmonics in the wave field environment usually indicates the appearance of additional energy, which in this case has brought the neutrino flux from the supernova. It should be noted that in [33] expected to impact and neutrinos, but without knowing the effect [12-13] There is interpretation was possible. However, the report had been made sufficiently "label" conclusions. Repeat it, adjusted for neutrino fluxes.

1). The precision measuring systems, reference and fundamental physics experiments to be considered Helios geophysics factors and modulated neutrino fluxes.

2). Gravitational - wave experiment in the framework of the Earth basically missed the coincidence circuit and accounting modulated neutrino flux renders meaningless.

$3)$. Register or manifestation of astrophysical effects of the scheme: the radiation and / or modulated beam of particles (neutrinos) from astrophysical sources to Earth as the grate antenna - seismic, electromagnetic, fluid dynamic, and so on. N. A response - the impact on astrophysical detectors (to search for neutrino fluxes - direct registration of radioactive detector).

4). Modern technical possibilities and space programs allow staging experiments to search for astrophysical effects of a coincidence circuit Earth - the Moon, the Earth - Mars, Mars - the Moon, etc.

\section{Conclusion}

Modern experiments on registration of modulated neutrino fluxes from astrophysical sources as well as review and retrospective data of processing earlier observations point to the registration of neutrino fluxes modulated and neutrino pulsed signal from supernova, pulsars, solar processes. It is available for the implementation of a focused neutrino telescope. And once again emphasize: Analysis of retro - as well as the results of future studies will possible only on the basis of the opening $[12,13]$.

\section{References}

[1] Sadeh D., Ben Menahem A., Meidav M. Possible detection of gravitational waves from pulsars: Preprint TAUP-270-72. 1972.

[2] Stanyukovich K. P, Khavroshkin O. B, Tsyplakov V.V. Detection of gravitational waves from pulsars / / Astronomer. circular. Bureau of Astronomical Communications of the USSR Academy of Sciences. 1974. № 824. 3 p.

[3] Pleskach N. K. Quasi-harmonic oscillations of the microseismic background in the frequency range $1-5 \mathrm{~Hz} / /$ Dokl. AN SSSR. 1977. Vol. 232, No. 3. P. 558-561.

[4] Samokhin A. A. The effect of laser radiation on absorbing condensed media, Tr. IOFAN. M., 1988. T.13.

[5] Gusev V. E., Karabutov A. A. Laser optoacoustics. M., 1991.

[6] O. Khavroshkin, V. Tsyplakov, Nonlinear Seismology: Space Component, Saarbrücken: Palmarium Academic Publishing, 2013, 516 pp., ISBN 978-3-659-98415-0

[7] Chamberlain J. Physics of auroras and radiation of the atmosphere. M.: I. L, 1963. 777 p.

[8] Elvey C. T. Aurora borealis // Adv. Electronics and Electron Physics. 1957. N 9. P. 1-42.

[9] O. Khavroshkin, V. Tsyplakov. Nonlinear seismoacoustics in cosmogony and astrophysics. RANS, Otd. Cosmogony. M. 2008, 48p.

[10] K. Shpiring. Neutrino high-energy astronomy: a glimpse of the promised land. Physics-Uspekhi, Vol.184, No. 5, 2014, pp.510-523.

[11] Oleg Khavroshkin, Vladislav Tsyplakov. RADIOACTIVITY OF NUCLEI IN A CENTRIFUGAL FORCE FIELD. The Natural Science (NS) Vol. 3, No.8, 733-737 (2011).

[12] Oleg Khavroshkin, Vladislav Tsyplakov. Sun, Earth, radioactive ore: common periodicity. The Natural Science (NS) Vol. 5, No. 9, 1001 -1005, (2013).

[13] Khavroshkin OB, Tsyplakov VV. Radioactivity, solar neutrinos, interactions. Engineering Physics.-2013, No. 8, pp 53-62.

[14] Khavroshkin OB, Tsyplakov VV Natural radioactivity as an open system. Engineering Physics.-2013, No. 12, pp.40-44.

[15] Nikolaev A. V., Khavroshkin OB, Tsyplakov VV. Structure of astrophysics component of seismic emission. Engineering Physics. 2016, No. 9, pp.69-73.

[16] Bullen, KE, Introduction to Theoretical Seismology. Moscow: Mir, 1966, 460p.

[17] Nikolaev AV, LN Rykunov, OE Starovoit, OB Khavroshkin, VV Tsyplakov. Nonlinear features of natural oscillations of the Earth. Academy of Sciences of the USSR, DNC, POI. Dynamic processes in discrete geophysical systems. Sat. works. Vladivostok, 1986, 138p.

[18] Lin'kov EM, Tipisev S. Ya. Observation of long-period oscillations of the Earth by a seismograph with a photoelectric converter. Academy of Sciences of the USSR, DNC, POI. Dynamic processes in discrete geophysical systems. Sat. works. Vladivostok, 1986, 138s. 
[19] Ness NF, J. Harrison, LB Slichter. Observation of the Earth's own oscillations.- In.: Own oscillations of the Earth. Moscow: Mir, 1954, 60-79 pp.

[20] Korchagin F. G., S. A. Chebotov. Some results of high-precision studies of deformation of the earth's surface. AN SSSR, DVO, TOI. Application of long-base laser interferometers in geophysics. Theses of reports, Vladivostok, 1987, 75p.

[21] Antonova LM, Savina NG Analysis of low-frequency oscillations of the Earth recorded by a laser deformograph. Ibid.

[22] A. V. Davydov, G. I. Dolgikh, W. H. Kopvillem, A. M. Zapolsky. The study of the background of the Earth's own oscillations. Part II. Ibid.

[23] O. Khavroshkin, V. Tsyplakov, Introduction to Nonlinear Seismology, Saarbrücken: Palmarium Academic Publishing, 2013, 400 pp., ISBN 978-3-659-98197-5.

[24] Man`ko V. I. (RAS). Reports at seminars of theoretical physics under the guidance of prof. AA Rukhadze. IOP RAS.

[25] Alan Smith, I. A. Crawford, Robert Anthony Gowen, R. Ambrosi, M. Anand, B. Banerdt, N. Bannister, N. Bowles, C. Braithwaite and P. Brown, et al. Experimental Astronomy. Volume 33, Numbers 2-3 (2012), 587-644, DOI: 10.1007/s10686-011-9250-5Original Article Lunar Net-a proposal in response to an ESA M3 call in 2010 for a medium sized mission.

[26] ATNF Pulsar Catalogue.
[27] O. Khavroshkin, V. Tsyplakov. Hydrogen maser: solar periodicity. Engineering Physics.-2014, No. 3, p. p.25-34.

[28] LV Grunskaya and co-authors, articles in the journal "Space, Time and Fundamental Interactions". April - June (2), 2014 Section "Part III. Identification of periodic components with frequencies of astrophysical processes in time series of the vertical component of the electric field strength of the boundary layer of the Earth's atmosphere ". www.profill.ru

[29] Khavroshkin OB, Tsarev VA, Tsyplakov VV, Chechin VA Interaction of neutrino beams with a seismically active medium. M., 1985. 16c. (Representative / FANSSSR, No. 167).

[30] Amaldi E., Coccia E., Frasca S. et al. Background of possible terrestrial origin. III.//Nuovo cim. C. 1989. Vol. 4, № 3. P. 299-308, 401-457.

[31] Ryazhskaya OG, Ryasny VG Letters in the ZhETF, 1988, 47, 236.

[32] Vavilov Yu. N., TZ Verbitsky, OB Khavroshkin. Analysis of the indications of the underground acoustic device on February 23, 1987. Letters to the JETP, vol. 50, no. 10, p. 411412. 1988, 47, 236.

[33] O. B. Khavroshkin, V. V. Tsyplakov. New problems of extraterrestrial seismology. Problems of geophysics of the XXI century. Sat. works in 2 books, Book 2, Managing editor, ch. - corr. RAS A. V. Nikolaev. RAS, United Institute of Physics of the Earth. O. Yu. Shmidt. M., "Science", 2003, pp.. 281-324. 